(Aus dem Institut für allgem. u. experim. Pathologie der Universität Wien.)

\title{
Ueber die Wirkung der Kalisalze auf das Herz und die Gefässe von Säugethieren.
}

\author{
Von \\ Privatdocent Dr. Ludwig Eraun.
}

(Mit 5 Textfiguren.)

Die ersten Untersuchungen über die Wirkung der Kalisalze auf.

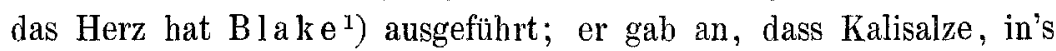
Blut injicirt, direct auf das Herz wirken und durch Herzstillstand den Tod herbeiführen können.

Nach ihm haben Bernard und Grandeau ${ }^{2}$ ) gelehrt, der rasche Tod nach Injection von Kalilösungen in die Jugularvene erfolge nicht durch Asphyxie, sondern durch Veränderung der Blutzusammensetzung; Herz und Lunge zeigen bei der Section völlig normalen Zustand.

Traube ${ }^{3}$ ) bezeichnet im Gegensatze zu Bernard und Grandeau die Wirkung der Kalisalze als jener der Digitalis gleich. "Wenn ich die Curven ansehe, vermag ich" - sagt er - „kaum einen Unterschied anzugeben . . . . Auch die Erscheinungen am Herzen sind vollkommen denen bei der Digitalis-Vergiftung gleich. Wenn der Tod durch Digitalis eingetreten ist, findet man bei sofortiger Eröffnung des Thorax das Herz vollständig bewegungslos. Lässt man jetzt die stärksten indirecten Ströme auf dasselbe einwirken, so vermag man auch nicht die Spur einer Contraction hervorzubringen, weder am ganzen Herzen noch an einzelnen Muskelbündeln, während man zur selben Zeit uud noch über ${ }^{1 / 2}$ Stunde lang an den einzelnen

1) Bl a ke e, Edinb. med. and. surg. journal. 1839.

2) Bernard und Grandeau, Journ., de l'Anat. et Physiol. t. 1 p. 378, cit. nach P. Guttmann, Berliner klin. Wochenschr. 1865.

3) Traube, Ges. Beiträge Bd. 1 S. 383. 
Körpermuskeln und selbst noch am Zwerchfell durch schwächere Ströme Zuckungen hervorrufen kann. Ganz so verhält sich das Herz, wenn der Tod durch Kalium nitricum eingetreten ist. - Kleine Dosen von Kalium nitricum bewirken eine Verminderung der Pulsfrequenz und Druckerhöhung; werden nun die Vagi durchschnitten, dann steigt die Pulsfrequenz enorm und gleichzeitig der Druck. - Auch bei der Prüfung der Wirkung des Kalium nitricum bei durchschnittenen Vagis zeigte sich das Gleiche wie bei Injectionen von Digitalis: Trotz der durchschnittenen Vagi vermindert sich bei Einspritzung einer kleinen Dosis die Pulsfrequenz unter steigendem Druck. Werden aber die Einspritzungen kurz nach einander wiederholt, so tritt keine Frequenzverminderung ein, während der Druck noch jedes Mal in die Höhe geht.

Auch Paul Guttmann ${ }^{1}$ ) hat in einer grossen Reihe experimenteller Untersuchungen den Beweis der Herzwirkung der Kalisalze erbracht, die nach ihm darin besteht, dass die Anzahl der Contractionen abnimmt und deren Energie geschwächt wird. "In kleinen Dosen setzen sie nur vorübergehend die Herzthätigkeit herab; wiederholte kleine Gaben haben cumulative Wirkungen und tödten; grosse Gaben führen rasch Herzstillstand herbei, während die Erregbarkeit und Functionsfähigkeit der Skelettmuskulatur noch lange erhalten ist. Ein mit Kalisalzen vergifteter Frosch führt bei vollständigem Stillstande des Herzens noch kräftige Muskelbewegungen aus.

Die Resultate Guttmann's stehen mit jenen von Traube im Einklang; auch er führt die verlangsamende Wirkung kleiner Gaben auf Vaguswirkung, die gleiche Wirkung vergiftender Kaligaben aber auf die Lähmung des musculo-motorischen Apparates im Herzen zurück, da das Herz nach solchen Vergiftungen auch trotz Vagusdurchschneidung und trotz Lähmung der Herzvagus-Endungen durch Curare zum Stillstande komme. Der Herzstillstand trete demnach ohne Betbeiligung der Vagi ein."

Unsere Kenntnisse von der Wirkung der Kalisalze auf den Kreislauf sind durch $\mathrm{Podcopaew}{ }^{2}$ ) gefördert worden, der die Ansicht aussprach, dass dieser Wirkung zum Theil eine Reizung der Vasomotoren zu Grunde liege, und einen Beweis dafür in der Milzverkleinerung erkannte, die nach der Einspritzung von Kalisalzen

1) Paul Guttmann, l. c.

2) Podcopaew, Virchow's Arch. Bd. 33.

E. Pflüger, Archiv für Physiologie. Bd. 103. 
in die Venen eintrat. Im Gegensatze zu Traube fand Podcopaew sodann eine Reihe von Verschiedenheiten zwischen Kalium- und Digitaliswirkung und zwar: Bei der Digitalisvergiftung werden die Herzbewegungen im Anfange sehr rasch, was er bei Vergiftungen mit Kalisalzen niemals beobachtet hat; bei Digitalisvergiftungen erfolgt der Herzstillstand in Systole, bei der Kaliumvergiftung in Diastole; die Digitalisvergiftung tritt ein, wohin immer das Gift injicirt wird, der Kalium-Herztod nur nach Injection der Salzlösung. in das Blut.

In seinen Untersuchungen „Ueber die physiologische Wirkung der Fleischbrühe" fand Kemmerich ${ }^{1}$, dass concentrirte Fleischbrühe in kleinen Dosen Zunahme der Zahl und der Stärke der Herzcontractionen, sowie Erhöhung des arteriellen Drucks, in grossen Dosen die Erscheinungen der Herzparalyse bewirke. Der Steigerung nach kleinen Dosen folge kein späteres Sinken des Pulses unter die normale Frequenz. - Das wirksame Princip der Fleischbrühe seien die Kaliumverbindungen.

$\mathrm{Bung} \mathrm{e}^{2}$ ) hat die Angaben von Kemmerich über den Einfluss des Fleischextractes auf die Herzthätigkeit nicht hestätigen können. $\mathrm{Er}$ hält die Annahme für gerechtfertigt, dass die nach subcutaner Injection kleiner Dosen von Kalisalzen eintretende Pulsbeschleunigung: bloss die Wirkung der Erregung durch den Schmerz sei; nach Injection von tödtlich wirkenden Dosen geht der Herzläbmung eine bedeutende Herabsetzung der Zahl und der Stärke der Herzeontractionen voraus.

Nach Aubert und Dehn ${ }^{3}$ ) erfolgt das Aufhören der Herzpulsationen nach Zufuhr tödtlicher Kalidosen wenige Secunden nach Beendigung der Injection, und zwar nicht ganz plötzlich, sondern nach vorherigem Sinken des Drucks, der Wellenhöhe und der Frequenz. Mitunter war auch noch eine geringe Drucksteigerung vor dem Eintritte des Absinkens zu sehen. Nachdem Pulslosigkeit eingetreten ist und 10-30 Secunden gedauert hat, folgen dann noch immer einige Pulsationen mit gleichzeitiger Erholung des Drucks, ohne dass es aber zu einer Wiedererholung kommt. Dagegen sieht man nach Dosen, welche der tödtlichen nahekommen, dieselbe Ab-

1) Kemmerich, Pflüger's Arch. Bd. 1 u. 4.

2) Bunge, Pflüger's Arch. Bd. 4.

3) Aubert und Dehn, Pfi üger's Arch. Bü. 9. 
nahme von Druck und Puls bis zum vollständigen Aufhören der Pulsationen; dann folgt aber ein sehr energischer Wiederbeginn der Herzthätigkeit, welche nach wenigen Secunden wieder bis zur Norm zurückkehrt. Bei kleinen Dosen und den ersten Injectionen überwiegt das Steigen des Drucks gegen die Drucksenkung; bei grossen Dosen und ofters wiederholten Injectionen herrscht die Drucksenkung vor. Wird das freiliegende Herz nach Zufuhr tödtlicher oder grosser, aber noch nicht tödtlicher Dosen direct beobachtet, dann sieht man an ihm Wogen und Flimmern sowie Peristaltik von der Spitze zur Basis und umgekehrt, von rechts nach links und umgekehrt. Diese Bewegungen haben keinen Effect für die Fortbewegung des Blutes. Diese Bewegungen können nun wieder aufhören und normale an ihre Stelle treten; das ist der Fall bei nicht-tödtlichen Dosen; es können geordnete Bewegungen auch bei tödtlichen Dosen auf einige Zeit wiederkehren; bald aber sinkt der Druck wieder ab, und es erfolgt weiter kein Zeichen von Herzthätigkeit.

Die Drucksteigerung und die Drucksenkung basiren nach Aubert und Dehn auf versehiedenen Herzwirkungen, denn das Steigen des Drucks erfolgt ohne Alteration der normalen Herzbewegung, das Sinken - wenigstens bei grossen Dosen - mit einer Störung derselben. Die zwei Wirkungen sind: Erregung des Herzens und des Herznervensystems und Störung der Coordination oder des Coordinationscentrums im Herzen. Die Kalisalze wirken nur auf das Herz, nicht auf die Blutgefässe ein. - Eine Cumulirung ihrer Wirkung lasse sich nicht nachweisen.

Eine gewisse Kaliumdosis muss nach den Erfahrungen der beiden Autoren als definitiv tödtlich angesehen werden, d. h. als Veränderungen erzeugend, welche nicht wieder aufgehoben werden können.

Im Gegensatze zu Aubert und Dehn fand Boehmi ${ }^{1}$ ), dass man durch Kalisalze vergiftete Herzen völlig wieder beleben könne. Er hatte gesehen, dass kleine Dosen Kalisalpeter $(0,05 \mathrm{~g}$ bei Katzen) zuerst eine Verminderung des Blutdrucks und der Pulsfrequenz von kurzer Dauer erzeugen, worauf der Blutdruck sich ziemlich rasch erhebt und Werthe bis $114 \mathrm{~mm}$ über die Norm erreicht. Während dieses Ansteigens ist die Pulsfrequenz nur wenige Secunden lang beschleunigt, während auf der Höhe der Blutdrucksteigerung constant eine erhebliche Pulsverlangsamung eintritt. Die

1) Boehm, Arch. f. exper. Pathol. u. Pharmak. Bd. 8. 
Vermehrung der arteriellen Spannung dauert höchstens 2-3 Minuten, worauf sich der Blutdruck wieder auf sein normales Niveau einstellt. Die Steigerung durch kleine Kalisalpeter-Dosen erfolgte auch nach vorausgegangener Halsmarkdurchschneidung. In grossen Dosen (von $0,1 \mathrm{~g}$ an) bewirkt das salpetersaure Kali keine Blutdrucksteigerung mehr, sondern tödtet das Thier unter rapidem Absinken des Blutdrucks. Die in completem diastolischem Stillstande sich befindenden Herzen solcher Thiere können nun wieder belebt werden. Das erste bei einer solchen Wiederbelebung wirksame Moment ist die Erneuerung des im Herzen stagnirenden Blutes - Verdünnung des Kaligehaltes - , das zweite der auf das Herz ausgeübte mechanische Reiz.

Die Angabe der Autoren, dass der Tod nach Kaliumvergiftung ein Herztod sei, ist noch einmal auf Widerspruch gestossen. Köhler ${ }^{1}$ ) nämlich betrachtet, eine secundäre Beeinflussung der Herzfunctionen durch Kalium nicht in Abrede stellend, als Ursache des Kaliumtodes nicht den factisch gar nicht vorhandenen Herzstillstand, sondern die Lähmung des vasomotorischen Centrums, welche unter plötzlicher Dilatation der peripheren Gefässe in maximo zum Absinken des Drucks auf den Nullpunkt Veranlassung gebe. Die zweite Ursache sei eine sehr starke, in Lähmung umschlagende Reizung des Athemcentrums. Thiere mit discudirtem Halsmark vertragen par. condit. grössere Kaliumdosen als unverletzte, weil ein Uebergreifen der Lähmung auf die peripher gelegenen Centren für die Gefässinnervation bis zu einem gewissen Grade so lange compensirt werden kann, als dank den fortbestehenden Contractionen des dem Einflusse der in der Medulla oblongata gelegenen Centren entrückten Herzens auch nur eine minimale Circulation fortdauert. Die Pulsverlangsamung führt Köhler auf die Blutdrucksteigerung zurück; das Absinken des arteriellen Drucks gehe mit Acceleration des Pulses Hand in Hand.

Die Untersuchungen von Mairet und Combemalle ${ }^{2}$ ) über die physiologische Wirkung des Kalisalpeters haben sich mit der Frage der Herzwirkung dieses Salzes nicht beschäftigt; auch $\left.\mathrm{Pugliese}{ }^{3}\right)$ ist in seinen Versuchen über den Einfluss von Koch-

1) Köhler, Centralbl. f. d. med. Wissensch. 1877.

2) Mairet und Combemalle, Soc. de biolog. 1887.

3) Pugliese, Arch. di farmak. e terap. 1895. 
salz und Chlorkalium auf den Stoffwechsel auf die Herzwirkung dieser Salze nicht eingegangen.

Hingegen fand Bottazzilis), dass isotonische Lösungen von Kalisalpeter, auf das suspendirte blutleere Froschherz aufgetropft, Verlangsamung der Schlagfolge und Verstärkung der einzelnen Systolen bewirken. Auf das in situ gelassene blutleere Herz wirkend erzeugt dieselbe Lösung Stillstand in Diastole. Hyperisotonische Lösungen erzeugen bald Schwächung und Stillstand des Herzens mitunter in Systole - , nach welchem Restitution durch Waschung mit alkalischer $0,75 \%$ iger Kochsalzlösung möglich ist. Auf das blutgefüllte suspendirte Herz tropfende Kalilösung erzeugt niemals Herzstillstand. - Bottazzi nimmt an, dass die Verlangsamung durch Vagusreizung zu Stande komme. -

Zuletzt hat noch, als meine Arbeit bereits abgeschlossen war, E. Gross') an dem mit Ringer'scher Flüssigkeit gespeisten Langendorff'schen Herzpräparate nachgewiesen, dass nach Injection von kleinen Kaliumdosen (KCl) Abnahme der Contractionsgrösse und Frequenz, bei grösseren Dosen diastolischer Herzstillstand erfolge, wobei die Erregbarkeit der Herzmuskulatur je nach der Menge des Kaliums herabgesetzt oder aufgehoben ist. Die Kaliumwirkung zeigte grosse Aehnlichkeit mit den Erscheinungen bei Vagusreizung, doch wurde bei letzterer, wenigstens am Ventrikel, niemals Unerregbarkeit der Muskulatur beobachtet. Von der Muscarinwirkung unterscheidet sich die des Kaliums dadurch, dass die letztere auch nach Atropininjection auftritt; ferner ist beim Muscarinstillstande der Herzmuskel erregbar. Bei der Application von Kaliumlösung auf den Vagus wurde, abgesehen von einer vorübergehenden Erhöhung der Erregbarkeit, nie eine Reizung des Vagus, sondern im Gegentheil - bei stärkeren Lösungen nach kürzerer, bei schwächeren nach längerer Zeit - eine Lähmung des Vagus beobachtet, die aber nach Ersatz des $\mathrm{KCl}$ durch 0,8\% \% ige NaCl-Lösung wieder schwand.

Bei Weglassen des Kaliums aus der Ring er'schen Flüssigkeit kommt es anfangs zu bedeutend vergrösserten und beschleunigten Contractionen, im weiteren Verlaufe aber bei unvollkommener diastolischer Erweiterung zur Verkleinerung der Herzcontractionen. -

1) Bottazzi, Arch. d. Physiol. Bd. 8 .

2) E. Gross, Pflïger's Arch. Bd. 99. 
Ueberblicken wir nunmehr die in der Literatur vorbandenen Angaben über die Wirkung der Kalisalze, so finden wir, dass ein solches Resumé eine Reihe von Widersprüchen ergibt und eine Reihe von Fragen zur Beantwortung übrig lässt.

Der Angabe von Blake über die Wirkung der Kalisalze auf das Herz steht die allerdings von allen anderen Autoren widersprochene Angabe Bernard's und Grandea u's gegenüber, der zu Folge der Kalitod nicht durch Asphyxie zu Stande komme. Traube hat die Herzwirkung der Kalisalze einwandfrei festgestellt und eine Analyse derselben versucht; er scheint dieselbe einerseits als Vaguswirkung, andererseits als directe Herzwirkung aufgefasst und auch eine Gefässwirkung der Kalisalze erkannt zu haben. Eine dem derzeitigen Stande unserer Kenntnisse entsprechende Beweisführung ist jedoch in seinen Auseinandersetzungen ebenso wenig zu finden wie in den Untersuchungen von Guttmann und Podcopaew. Die Resultate Kemmerich's und Bunge's stehen einander diametral entgegen; die Angabe von Aubert und Dehn, dass die Kalisalze nur auf das Herz, nicht auf die Blutgefässe und ihre Nerven, einwirken, ist mit den Befunden von Traube und Podcopaew in Widerspruch, und die Angabe von Boehm, dass man durch Kalisalze vergiftete Herzen wieder beleben könne, harrt noch der Bestätigung. Die Ansicht Köhler's, der Kalitod komme durch Lähmung des vasomotorischen Centrums zu Stande, ist bisher vereinzelt geblieben, und die letzten der Kaliwirkung gewidmeten Untersuchungen, die E. Gross ausgeführt hat, konnten, dem Versuchsplane entsprechend, unseren Gegenstand nicht erschöpfen.

Da man erwarten durfte, an der Hand der uns jetzt zur Verfügung stehenden physiologischen Untersuchungsmethoden unsere Kenntnisse von der Kaliumwirkung auf Herz und Kreislauf erhärten, viellejcht auch erweitern zu können, habe ich mit gütiger Erlaubniss des Herrn Professors Paltauf im Laboratorium desselben eine Reihe von einschlägigen Versuchen ausgeführt.

Die Versuche sind an Kaninchen, Katzen und Hunden mittelst der Methode der Blutdruckschreibung, nach der Suspensionsmethode mit und ohne Blutdruckschreibung, am Hering-Bock'schen und am Langendorff'schen Präparate ausgeführt worden.

Beschreibungen der angewandten Methoden sind in diesem Archive schon so bäufig wiedergekehrt (B. 61, 73, 82, 93, 99 etc.), dass ich dieselben mit den entsprechenden Hinweisen unterlassen darf. 
Meinen Ausführungen liegen die Resultate von 28 gelungenen Versuchen zu Grunde.

Da sämmtliche (anorganischen) Kalisalze die nämliche Wirkung auf Herz und Kreislauf ausüben ${ }^{1}$ ), darf ich, obwohl ich nur mit $\mathrm{KNO}_{3}$-Lösungen gearbeitet hate, von der Wirkung der Kalisalze im Allgemeinen sprechen.

Injicirt man einem Kaninchen ganz geringe Mengen $(0,005-0,01)$ einer Kalisalzlösung ${ }^{2}$ ) in die Jugularvene, dann findet man, als Folgen dieser Injection, eine Blutdrucksteigerung geringen Grades mit oder ohne gleichzeitige Frequenzvermehrung des Herzschlags, welch' letztere jedoch nur in den selteneren Fällen verzeichnet wird, während die geringe Drucksteigerung unter den erwähnten Bedingungen als ziemlich regelmässiger Befund zu notiren ist.

Nach Zufubr etwas grösserer Dosen $(0,01-0,2 \mathrm{~g})$ wird die Drucksteigerung von einer vorübergebenden Senkung des Drucks abgelöst, der eine zweite Steigerung auf dem Fusse folgt. Je grösser die erste Drucksteigerung, desto geringer ist die ihr nachfolgende Drucksenkung. Dieses Verbältniss gilt für die Senkung und die zweite Steigerung nicht.

Eine Dosis, die als gross zu bezeichnen ist, weil eine minimale Mehrgabe unter Umständen bereits eine schwere Schädigung des Herzens, ja sogar definitiven Druckabfall bewirken kann (circa 0,04 g), verändert das Bild der Wirkung wesentlich. Wir finden dann eine oft recht beträchtliche Senkung, bierauf eine Drucksteigerung, die bis an $100 \mathrm{~mm} \mathrm{Hg}$ und darüber betragen kann. Senkung und Steigerung gehen ohne deutliche Frequenzänderung einher.

Wir können also eine primäre und eine secundäre Drucksteigerung unterscheiden. Nach Zufuhr der für ihre Production erforderlichen Dosen tritt - wie erwähnt - während der primären Drucksteigerung eine wesentliche, in Betracht ziehbare Frequenzänderung (dann immer Vermehrung) nur selten ein. Eine Frequenzvermehrung während der secundären Drucksteigerung konnte niemals beobacbtet werden. Die primäre Drucksteigerung ist gering, die secundäre oft sehr bedeutend.

1) Aubert und Dehn, l. c.

2) In Bezug auf die Dosirung ist den Angaben von Aubert und Dehn nichts Neues hinzuzufügen. - Wir haben bei den Kaninchenversuchen stets mittelgrosse Thiere von circa $1 \mathrm{~kg}$ Gewicht verwendet. 
Ludwig Braun:
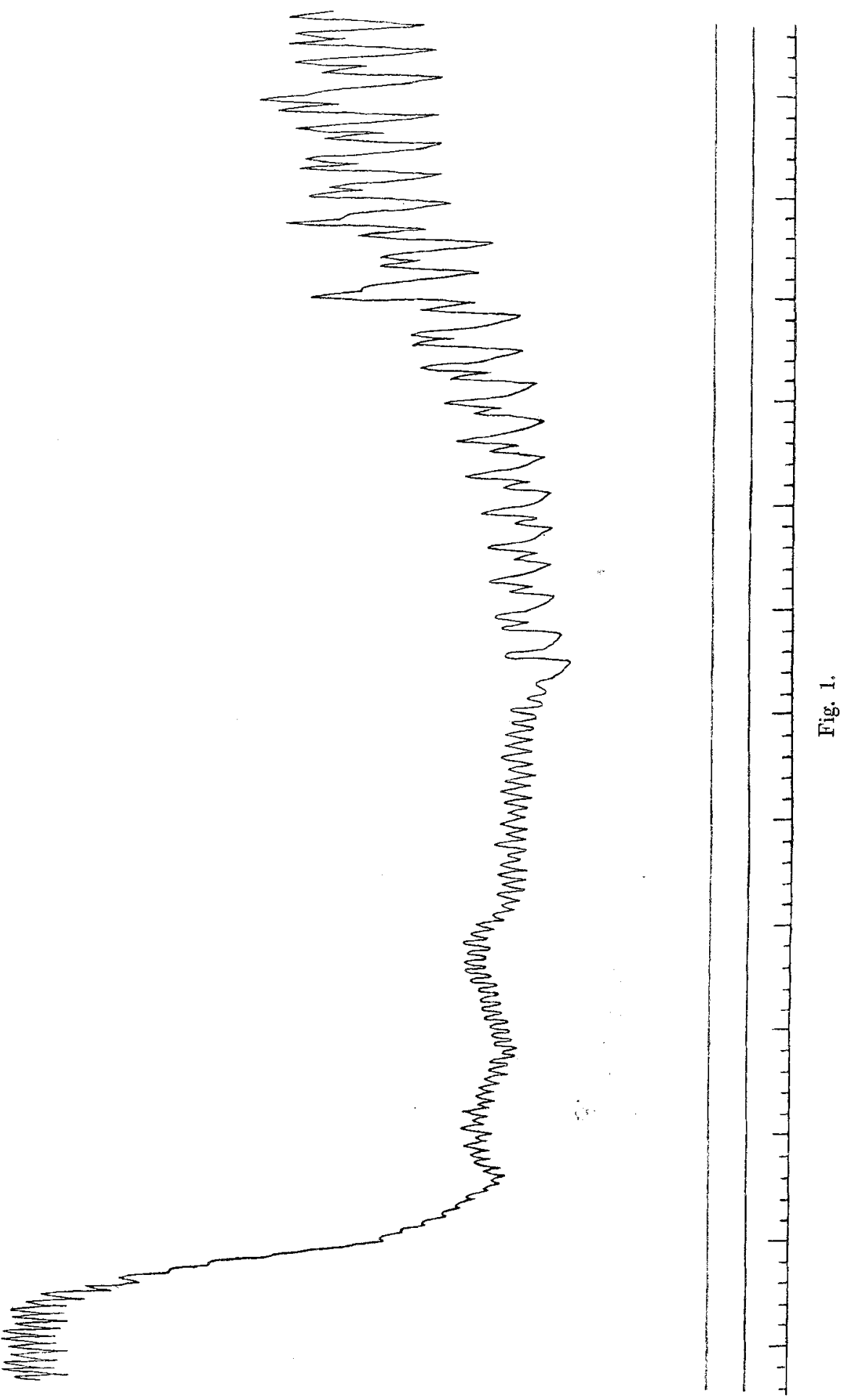
Einer fast letalen Dosis folgt eine tiefe Senkung mit oder ohne darauf folgende geringe Steigerung neben Frequenzabnahme; die primäre Steigerung fehlt gänzlich. Der Wiederanstieg wird von einer Reihe grosser Schwankungen vorbereitet, die Vaguspulsen zu gleichen scheinen (Fig. 1).

Letale Dosen sind von einem rasch (innerhalb einer bis mehrerer Secunden, je nach der Höhe der Dosis) eintretenden Druckabfall gefolgt; in ganz seltenen Fällen kann auch der definitiven Senkung noch eine kleine Steigerung vorangehen oder die horizontale Linie, die der Schreiber nach dem Curvenabfalle zieht, von mehreren kleineren und grösseren Schwankungen unterbrochen sein.

Ist das Herz blossgelegt, dann sieht man, dass den Drucksenkungen eine Blähung des Herzens entspricht. Der Grad dieser Blähung geht dem Ausmaasse der Senkung parallel, die zu gleicher Zeit von dem Druckschreiber verzeichnet wird. Geringere Senkungen scheinen ohne gleichzeitige Volumsänderungen des Herzens einhergehen zu können, nicht umgekehrt, d. h. einer Blähung des Herzens entspricht jedes Mal eine Senkung der Blutdruckkurve.

Während der Zeit und kurz nach einer rasch vorübergehenden, geringgradigen Blähung verzeichnet der Blutdruckschreiber bisweilen ziemlich beträchtliche Schwankungen.

Mittelst der Suspensionsmethode kann man sich sodann davon überzeugen, dass diese Blähung fastimmer an allen vier Herzabtheilungen zugleich einsetzt.

Während einer geringeren secundären Drucksteigerung pflegt die von den Schreibern verzeichnete Amplitude (an allen vier Herzabtheilungen) geringer zu werden.

Es kann vorkommen, dass die Blähung am linken Vorhofe und am linken Ventrikel früher auftritt als an den übrigen Herzabtheilungen, doch gibt es auch Umkehrungen in diesem Verbalten; einmal ist - was vielleicht notirt zu werden verdient - der Conus der Pulmonalarterie der zuerst geblähte Herztheil gewesen. Bisweilen schien es auch, als ob durch kleine Dosen der rechte Ventrikel vor dem linken betroffen werden könnte.

Die bedeutenden Drucksenkungen (und Blähungen) sind immer von bedeutenden Pulsverlangsamungen begleitet; den kleinen Senkungen entspricht zumeist keine Frequenz- 
änderung. Niemals ist die Blähung von Pulsvermehrung begleitet.

Nach Zufuhr letaler Kaliumdosen stirbt das Herz zumeist flimmernd $a b$.

Die Thatsache, dass die Blutdrucksenkungen nach Zufuhr kleinster Kaliumdosen fehlen, dass sie ohne Frage in geradem Verhältnisse zur Grösse der Dosis stehen, und dass sie auch je nach der Intensität der gleichzeitig eintretenden Herzblähung geringer oder grösser werden, lässt uns vermuthen, dass wir sie als $A u s d r u c k$ einer Schädigung der Herzleistung aufzufassen haben.

Eine solche Schädigung der Herzleistung könnte zu Stande kommen durch primäre Schädigung des Herzens oder auf secundärem Wege, durch Beeinträchtigung der Vasomotoren, Veränderung der Füllung des Herzens.

Eine primäre Schädigung kann wiederum durch Vagusreizung, die einen "hypodynamen Zustand" des Herzens herbeiführt ${ }^{1}$ ), oder durch directe Herzwirkung entstehen. Hat sie ihren Ursprung in einer Vagusreizung, dann müsste sie durch den Effect einer Acceleransreizung beseitigt werden können. Wir wissen ja seit den Untersuchungen von $\mathrm{R}$ oy und $\mathrm{Adami}{ }^{2}$ ), dass der Umfang von Vorhofs- und Kammercontraction bei Acceleransreizung in den meisten Fällen zunimmt. Die Zunahme dieser Contractionsgrösse steht in keinem bestimmten Verhältnisse zur etwaigen Beschleunigung und tritt auch ein, wenn die Herzfrequenz durch die Reizung fast gar nicht beeinflusst wird. $\mathrm{Zu}$ demselben Ergebnisse sind B ayliss und Starling ${ }^{3}$ ) gelangt. Speciell beim Hunde fand auch Franck ${ }^{4}$, dass Acceleransreizung in der Regel die Contractionsenergie der Vorhöfe und der Ventrikel steigert. Die nämlichen Erscheinungen hatten Heidenhain, Gaskell und Mills ${ }^{5}$ ) am Kaltblüter schon vorher nachgewiesen.

Acceleransreizung hat den Effect der Kalisalzzufuhr nicht zu beseitigen vermocht.

Die directe Schädigung des Herzens dureh Kaliumzufuhr erhellt sodann aus den übrigen Folgen der Injection, welche,

1) F. B. Hoffmann, Pflüger's Arch. Bd. 84.

2) Cit. nach Tigerstedt, Physiologie des Kreislaufes.

3) Journ. of physiol. Bd. 13 .

4) Arch. de physiol. 1890.

5) Cit. nach Tigerstedt, 1. c. 
je nach der Grösse der Kaliumdosis, als Tonusschwankungen, Blähung, Incoordination der Herzthätigkeit, Dyschronismus der Schläge der Herzabtheilungen, Antiperistaltik, in die Erscheinung treten, ferner aus dem Umstande, dass die nämlichen Folgen, in völlig unveränderter Weise, am atropinisirten Herzen zu verzeichnen sind. Die scheinbaren Vaguspulse sind also durch Arythmien und zeitweise auftretende Retardationen verursacht.
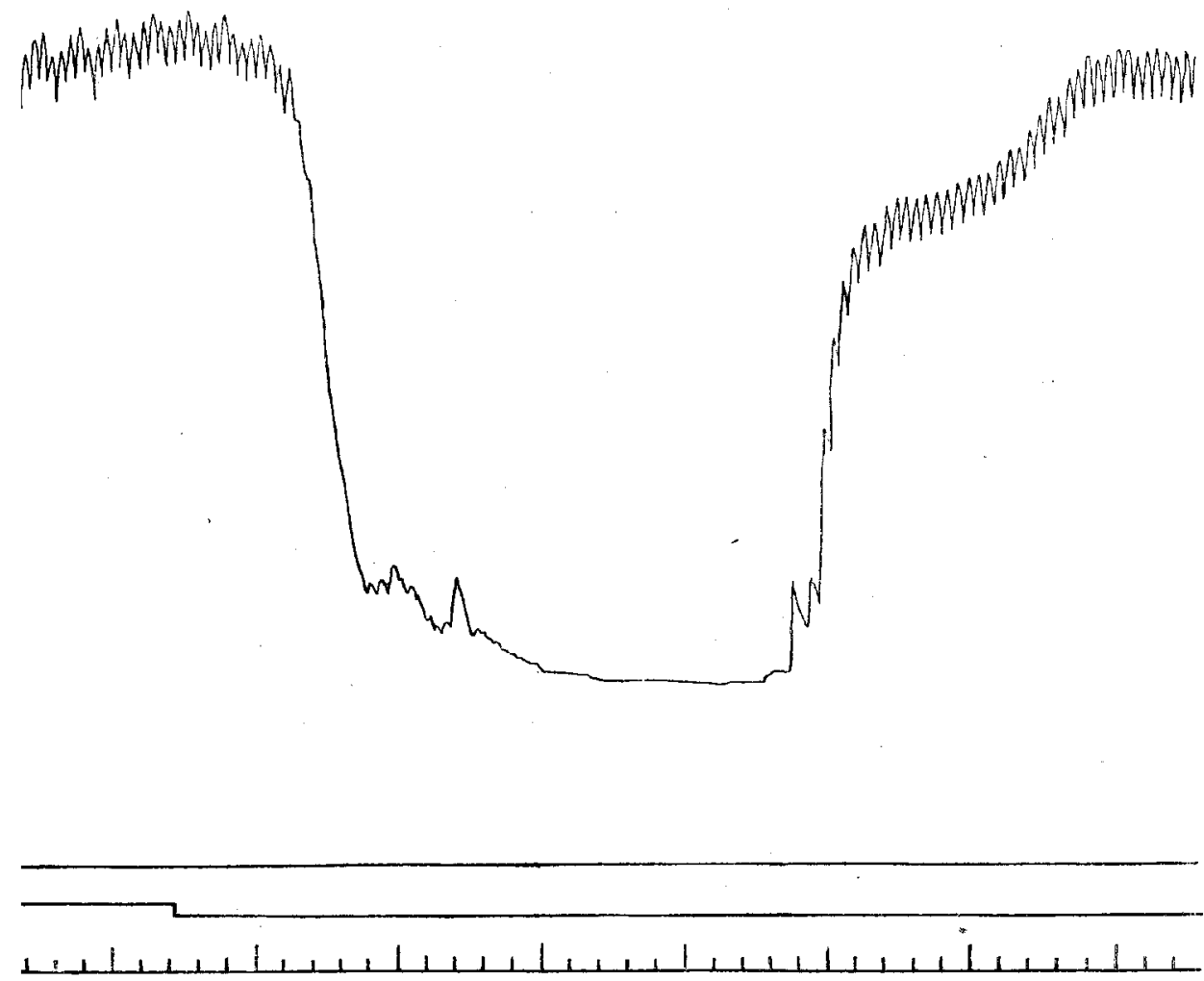

Fig. 2.

Auch die Versuche am Hering-Bock'schen Präparate lehrten mich, dass die Blutdrucksenkung als Ausdruck einer directen Herzschädigung aufgefasst werden muss (Fig. 2).

Nachdem ich also erkannt hatte, dass es sich bei der Kaliumwirkung um eine directe Herzwirkung handle, habe ich mich dem Studium derselben am Langendorff'schen Präparate zugewandt. Bei diesen Versuchen hat sich mir neben der Bestätigung der bisher erhobenen Befunde u. A. die bemerkenswerthe 
Thatsache ergeben, dass es nicht möglich ist, ein Herz durch Kaliumzufuhr zu tödten, wofern man nur dafür Sorge trägt, dass die Giftlösung baldigst wieder aus dem Herzen entfernt wird. Ich komme hierauf nochmals zurück.

Nun war zu untersuchen, welcher Antheil der Kaliumwirkung eventuell auf eine Gefässwirkung $\mathrm{zu}$ beziehen sei. Dabei greife ich wieder auf frühere Versuche zurück. Wir fanden Senkung nach Zufuhr mittlerer Dosen und eine erst nach der Senkung eintretende Steigerung des Blutdrucks, sahen, dass bei grösseren Dosen die Senkungen immer beträchtlicher werden und die Drucksteigerungen immer mehr in den Hintergrund treten, fanden schliesslich, dass nach letalen Dosen keine Steigerung des Drucks mehr eintritt, der Schreiber vielmehr bis auf die Abscisse herabsinkt und das Herz in maximal geblähtem Zustande stillesteht.

Es war von vorneherein wahrscheinlich, dass es sich hier um zweierlei Wirkungen handle, eine Herzwirkung und eine Vasomotorenwirkung.

Das Resultat der Versuche am Hering-Bock'schen Präparate machte uns dies ja wahrscheinlich, da die dabei erhaltenen Curven nach Zufuhr grösserer und ganz grosser Dosen jedwede Blutdrucksteigerung vermissen lassen (Fig. 2).

Um die beiden Componenten der Kaliumwirkung - die Herzwirkung und die Vasomotorenwirkung - ganz einwandfrei feststellen und auseinanderhalten zu können, habe ich nach dem Vorschlage Dr. Rothberger's den folgenden Vorgang gewählt: Die Giftlösung wurde herzwärts in die Carotis injicirt.

Hierbei wurde angenommen, dass die Kaliumlösung "zunächst nicht in's Herz gelange, sondern, durch das vorbeiströmende Aortenblut mitgerissen, den Kreislauf passiren müsse, ehe sie in das Herz einfliesst. Controlversuche mit einer concentrirten Methylenblaulösung belehrten uns über die Richtigkeit dieser Annahme ${ }^{1}$ ). Nach herzẉärts in die Carotis einverleibter Injection ist, sofern nicht eine letale Dosis zur Anwendung gelangt, die Thatsache zu

1) Injicirt man in die Carotis herzwärts eine starke Methylenblaulösung, dann sieht man die Blaufärbung in der Cruralarterie früher erscheinen als in den Coronargefässen; erst nach einigen Secunden wird das Herz blau, und zwar deutlich zuerst der rechte Ventrikel. 
registriren, dass die Drucksenkung ausfällt und nur die Drucksteigerung bestehen bleibt (Fig. 3 und 4).

Das Fehlen der Drucksenkung ist darauf zurückzuführen, dass die injicirte Kaliummenge nunmehr, mit dem circulirenden Blute vermischt und entsprechend verdünnt, keine wesentliche Wirkung mehr auf das Herz ausübt, während die in ungeschwächter Weise zu Stande kommende Drucksteigerung auf die Constriction der Gefässe $\mathrm{zu}$ beziehen ist.
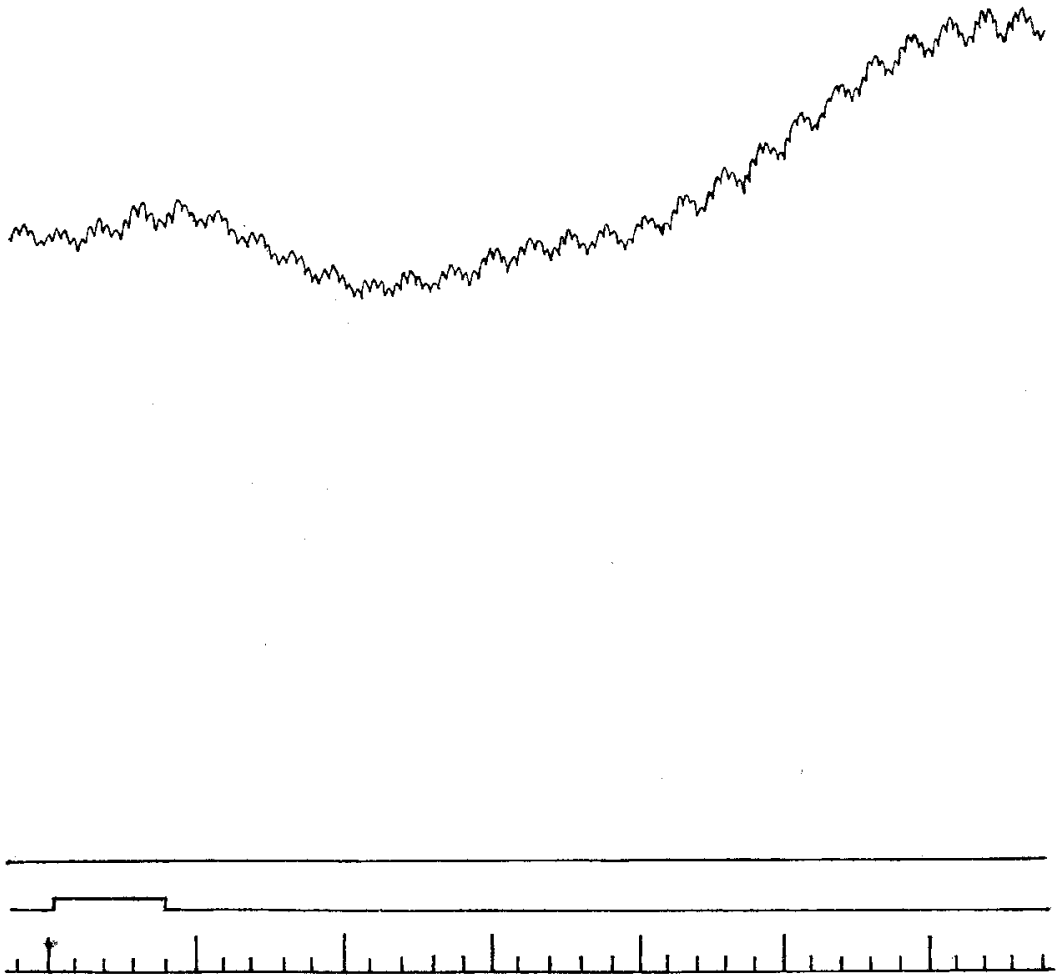

Fig. 3.

Um zu entscheiden, ob diese vasoconstrictorische Wirkung central oder peripher ausgelöst wird ${ }^{1}$ ), habe ich auch nach Ligatur der Hirnarterien eine herzwärts in die Carotis gerichtete Injection von Kaliumnitrat vorgenommen. Ich bin dabei zu dem gleichen Resultate gelangt und muss also annehmen, dass es sich um eine peripher ausgelöste Vasomotorenwirkung handelt.

1) Es konnte ja ein Theil der Lösung sofort in die zweite Carotis und hirnwärts getrieben worden sein. 
Die secundäre Drucksteigerung bleibt bestehen, solange das Herz selbst durch die Giftlösung ungeschädigt bleibt und den grösser gewordenen Widerständen eine entsprechende Mehrleistung entgegenzusetzen vermag.

Grössere, das Herz schädigende Dosen führen neben der Vasomotorenwirkung eine directe Herzschädigung herbei, die sich $u$. A. in Form von Pulsverlangsamungen und Verzögerungen der Wiedererschlaffung (Fig. 5) äussert, von denen die geringergradigen Druck-
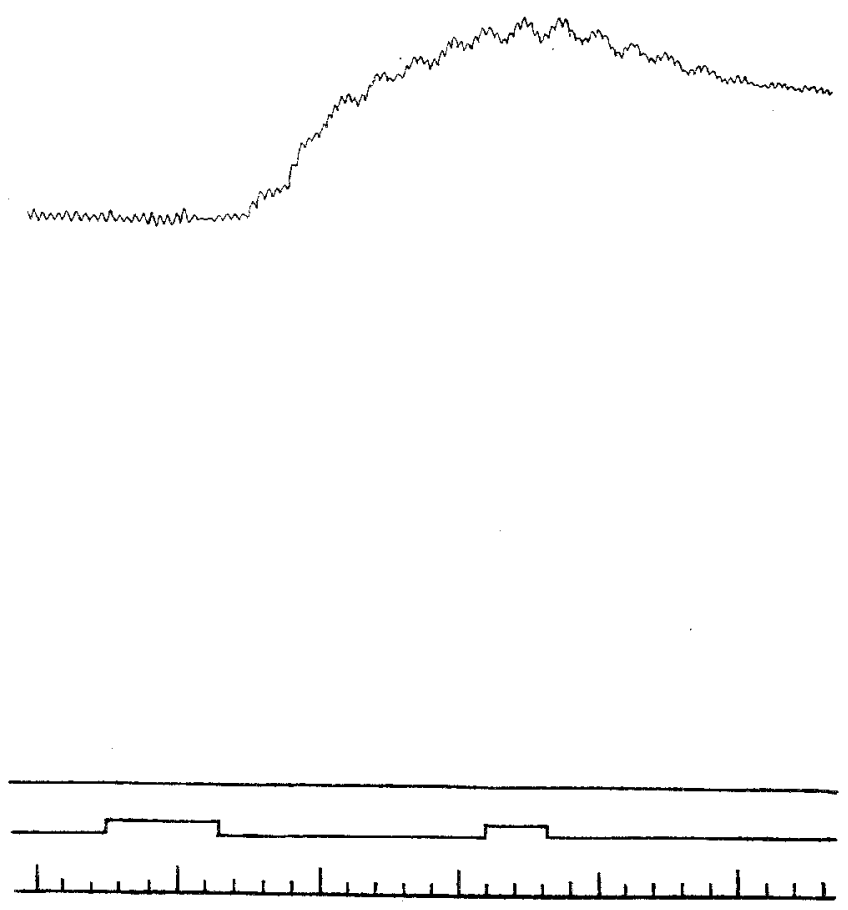

Fig. 4.

steigerungen begleitet zu sein pflegen. Grosse, das Herz schwer schädigende Dosen, in deren Gefolge die Herzthätigkeit nicht bloss wesentlich verlangsamt, sondern auch antiperistaltisch oder incoordinirt wird, lassen auch eine secundäre Drucksteigerung vermissen, da die schwere Schädigung dem steigenden Gefässtonus gegenüber natürlich ehestens hervortreten muss.

In der primären Drucksteigerung mit der sie bisweilen begleitenden Pulsvermehrung konnte man nach vielfachen Analogien eine directe Reizwirkung auf das Herz, eine Erhöhung der Herz- 
leistung, vermuthen. Das Resultat der Versuche am HeringBock'schen Präparate scheint diese Annahme immerhin zu bestätigen; jedenfalls aber ist die die Herzarbeit begünstigende Componente der Kaliumwirkung als recht unbedeutend zu bezeichnen.

Nunmehr wäre noch eine Beobachtung zu er-

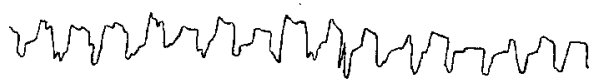
wähnen, die bereits angedeutet worden ist, und die schon $\mathrm{Boehm}{ }^{1}$ ) in zutreffender Weise geL. Atr. schildert hat. Es ist

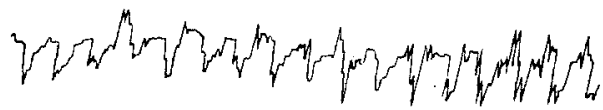
L. Ventr. dies die Möglichkeit der Wiederbelebung von durch K-Salze vergifteten Herzen. Boehm gab an, dass zur Restitution der Herzthätigkeit zwei Momente wirksam sein müssen: die Verdünnung des Kaligehaltes und ein auf das Herz ausgeübter mechanischer Reiz. Thatsächlich ist am überlebenden Herzen (Langen -

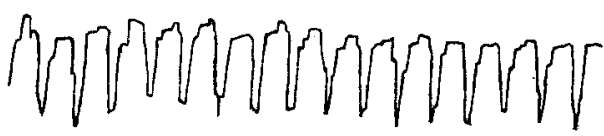

R. Atr.

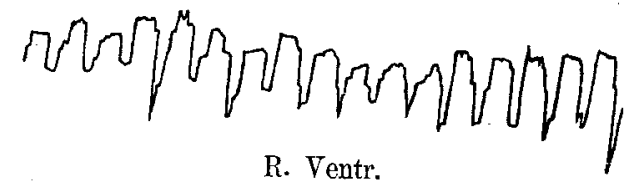

R. Ventr. d orff-Präparate) leicht der Beweis dafür zu erbringen, dass die vollständige Wiederherstellung der Herzthätigkeit

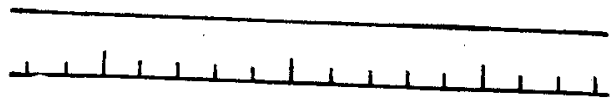

Fig. 5 . immer gelingt, auch wenn nach Einverleibung grösster Dosen $\left[1 \mathrm{~g}\right.$ und mehr $\left.\left.{ }^{2}\right)\right]$ bereits regellose Arythmie oder selbst mehrere Minuten dauernder Herzstillstand eingetreten ist; nur muss man dafür sorgen, dass die Giftlösung, eventuell unter höherem als dem bei den Versuchen gewöhnlich verwendeten Drucke, aus den

1). 1. c.

2) Die durchschnittliche letale Dosis für Kaninchen beträgt $0,05 \mathrm{~g}$. 
Coronargefässen und dem Herzmuskel wieder herausgewaschen wird. Die Massage des blossgelegten Herzens ist jedoch zu seiner Restitution nicht nothwendig. Es ist mir ein Mal gelungen, ein Herz, das lange stillgestanden hatte, durch blosse Injection von Kochsalzlösung in die Jugularvene wieder zu kräftigem Schlagen zu bringen, ja, ich sah sogar eine erloschene Herzthätigkeit wiederkehren, nachdem mittelst einer Pravaz'schen Spritze einige Kubikcentimeter Kochsalzlösung durch die Herzwand hindurch direct in den Ventrikel injicirt worden waren, was allerdings bei dem jedesmaligen Einstechen auch einen erneuten mechanischen Herzreiz bedeutet hat. -

Wir haben also gefunden, dass die Kalisalze nach intravenöser Application Herz und Kreislaufin zweifacher Weise zu beeinflussen vermögen: durch directe Herzwirkung und durch Vasomotorenwirkung (Vasoconstriction).

$\mathrm{Nur}$ in kleinsten Dosen scheinen die Kalisalze eine - übrigens kaum nennenswerthe - stimulirende Wirkung auf den Herzmuskel ausüben zu können. Einigermaassen grössere Kaliumdosen führen bereits Schädigungen, beziehungsweise deletäre Beeinträchtigungen, des Herzens herbei; diese Wirkungen sind durch Entfernung des Kalisalzes aus dem Herzen prompt zu beseitigen. Von einer vasodilatatorischen Wirkung der Kalisalze kann nach unseren Erfahrungen keine Rede sein.

Die therapeutische Verwendung der Kalisalze als Herzstimulantien erscheint nach meinen Befunden kaum berechtigt; sie kommen bloss als "Vasomotorenmittel" in Betracht.

Ich erlaube mir, dem Vorstande des Instituts, Herrn Professor Paltauf, für die Ueberlassung der Institusbehelfe, Herrn Professor $\mathrm{Bi}$ ed l für das meiner Arbeit jeder Zeit entgegengebrachte Interesse und Herrn Assistenten Dr. Rothberger für die Unterstützung bei meinen Versuchen meinen ergebenen Dank auszusprechen. 